Research Article

\title{
Water Dragon Boat Training Monitoring System Based on Multisensor Data Fusion Technology
}

\author{
Heng Shen \\ School of Physical Education, Huazhong University of Science and Technology, Wuhan, 430074 Hubei, China \\ Correspondence should be addressed to Heng Shen; 2017210210@hust.edu.cn
}

Received 13 August 2021; Revised 8 October 2021; Accepted 26 October 2021; Published 18 November 2021

Academic Editor: Mu Zhou

Copyright (C) 2021 Heng Shen. This is an open access article distributed under the Creative Commons Attribution License, which permits unrestricted use, distribution, and reproduction in any medium, provided the original work is properly cited.

\begin{abstract}
With the development of science and technology, a variety of electronic devices have entered our lives, making our lives more intelligent and making our work more effective. This article is aimed at studying the application of multisensor data fusion technology to the water dragon boat training monitoring system. In that case, we can analyze the various physical indicators of dragon boat athletes based on the data reflected by these sensors, when they can reach their physical limits and can perform in the best state to obtain the best results. The sensor is used to decompose the relevant data of each part of the athlete's limbs. This step is based on the image and understands the maximum value of the data to adjust the training goal. This article proposes some data fusion algorithms, using Kalman filter method, Bayesian estimation method, and DS evidence theory algorithm to compare data fusion systems, through the comparison to find the best fusion accuracy, and then get the most suitable method is then applied to this water dragon boat monitoring system to enhance the training efficiency of dragon boat athletes. The experimental results in this paper show that when the value of the parameter increases from 0.97 to 2.5 , the average classification accuracy of the $k$-NN classifier decreases from 0.97 to 0.4 , and the accuracy of the fusion results of the three fusion rules is also reduced correspondingly, but in this paper proposed, RP fusion rule still has better performance than the other two fusion rules. When the classifier is $k-\mathrm{NN}$, the three fusion rules increase with the number of sensors, and the accuracy of the fusion results is correspondingly improved. However, the final fusion accuracy obtained by the RP fusion rule proposed in this paper is always better than NB integration rules, and WMV integration rules are higher. Through these analyses, a training program that is most suitable for dragon boat athletes can be worked out, so that the athletes will not be useless. Multisensor data fusion technology brings great convenience to water dragon boat training and can provide more reasonable and accurate data to explore a practical way on the basis of ensuring the safety of personnel.
\end{abstract}

\section{Introduction}

1.1. Background. Competitive water boat sports are a traditional sport that perfectly combines entertainment and competition. With the development of science and technology, the stimulation of economic interests, and the drive of national culture, competitive sports have developed rapidly. In the modern competitive sports arena, competition has become more intense, which places higher demands on athletes' physical fitness. The water dragon boat started in the Spring and Autumn Period and the Warring States Period and has a history of more than 2,000 years. The water dragon boat activity is easy to hold in the south because it is more suitable for natural conditions. The natural environment in the north is limited, and the vast waters are lacking; so, the dragon boat dance in the dry land came into being. Although it does not have the surging momentum of the water, it also gives people a refreshing feeling, making the North Dragon Boat Festival atmosphere stronger. In recent years, multisensor data fusion technology has been widely used in both military and civil fields. Multisensor fusion technology has become a concern in many aspects such as military, industrial, and high-tech 
development [1]. With the rapid development of electronic information technology, various large-scale electronic systems continue to emerge, and their application backgrounds continue to become more complicated. The realization of their functions requires the support of various multisensor data systems. Therefore, it is necessary to support various sensors and different information source for more effective integration. At the same time, with the development of modern warfare, the role of multisensor data fusion technology in the military has become more prominent. It combines the characteristics of a variety of different sensors to obtain different types of target information from multiple directions and angles and improve the system in all dimensions. The coverage of the above improves the detection and recognition capabilities of the target. After years of development, multisensor data fusion technology has made considerable progress. In terms of fusion level, three levels are formed: data-level fusion, feature-level fusion, and decision-level fusion. In terms of system structure, centralized, decentralized, distributed, and hybrid structures are proposed; in terms of fusion algorithms, algorithms such as weighted average method, neural network method, Kalman filtering method, Bayesian estimation method, and D-S evidence theory are formed. In terms of application, multisensor data fusion has been widely used in military and civilian fields and has achieved remarkable results.

1.2. Significance. For wireless sensor networks, collaboration and real-time monitoring can be achieved, and the collected information can be processed and transmitted to users. It can be realized through information technology based on our real world cognition, thus effectively changing the way of information interaction between human beings and nature. We are fully able to use wireless sensor-based network technology to recognize things in the world, as far as possible to expand the existing network functions and human cognition of the world. With the continuous and rapid development of sensor technology, wireless communication technology, and computer technology, the use of sensor networks in environmental monitoring has also become a reality. Most of today's environmental monitoring systems use wired monitoring methods. Therefore, this kind of system generally has two problems: one is that the wired monitoring system is more dependent on the line; so, the layout of the system will inevitably be affected by the wiring [2]. The second is that the distribution of wired nodes is relatively fixed. Once some of the nodes have problems, it will inevitably lead to the loss of the monitoring function in a local area. This article uses a wireless sensor network to collect ambient temperature and humidity and transmits the collected information to the monitoring center in time. Compared with traditional monitoring methods, the use of wireless sensors has the following obvious advantages: (1) the network nodes have a higher distribution density, and each node involved can monitor the information in the local environment and transmit it to the data center; so, it has the characteristics of large amount of data collection and high precision. (2) The sensor node has a small volume, and the entire network only needs to be deployed once. (3) The wireless sensor node itself should have good computing capabilities, storage capability, and wireless communication capabilities and be able to perform more complex monitoring.
1.3. Related Work. After reading a lot of related literature, I found that multisensor data fusion technology is applied in many fields. With technological breakthroughs, we have more extensive applications. In Zhang's research, their team proposed a method to use redundant information to fuse spatial data and multimedia information in a multimedia sensor network. Experimental results show that their proposed method can reduce the energy consumption of spatial data and multimedia information fusion in multimedia sensor networks, and the fusion accuracy is high. Although their application prospects are very good, there are still certain technical barriers [3]. In Wu research, a path design method based on ant colony optimization (ACO) algorithm is proposed, which can improve the accuracy and effectiveness of data. However, it was not considered comprehensively in the experiment, and some factors were not taken into account. Aiming at the problem of PID controller parameter optimization design, the results of ant colony algorithm design and genetic algorithm design were compared. Numerical simulation results show that the ant colony algorithm has the effectiveness and application value of a new simulation evolution optimization method. However, this is just the emergence of simple rules [4]. In Zhang's research, artificial intelligence immune agents are proposed and applied to solve the problems of low data accuracy and delay caused by dynamic environmental changes. The experimental results show that the proposed method can improve the performance of the system by using this method. However, in this study, the results of the study were not so accurate. When he conducted the experiment, many environmental factors were not taken into consideration, which led to the lack of rigor of the results [5]. In Ma's research, a mileage pile positioning technology based on multisensor information fusion is proposed. This can avoid the cumulative error per kilometer using GPS and road environment video. The gyroscope is used as a substitute when the GPS signal is lost and is also used to correct wobble errors. The practical value of his experiment is very high, but there are still certain technical barriers [6]. In Xu's research, his research results show that the clustering fitting effect is as high as $99.83 \%$, which proves that the multiattribute DBSCAN algorithm and clustering analysis algorithm have higher reliability and provide better theoretical guidance for the analysis of abnormal ship behavior. Although the research prospects are perfect due to technical defects, the current results are still not so perfect [7]. In Zhang's research, a cooking production safety monitoring and early warning system based on information fusion was proposed. To achieve the goal of accurate monitoring of safety conditions and reliable early warning of dangerous situations, the security information of the monitoring and early warning area is collected through a sensor network such as combustible gas sensors, dust sensors, temperature sensors, pressure sensors, flow meter, and IP cameras, to establish security monitoring and early warning information fusion The model is then used to fuse various safety information through BP neural network and DS evidence theory algorithms. This research is of great practical value, but there are certain limitations [8]. In Cui's research, their team proposed a new program based on Hilbert-Huang transformation and deep learning for network attack detection in direct current (DC), microgrid (MG), and distributed power 
generation (DG) attack detection unit and its sensors. A deep learning method for advanced elective group using krill swarm optimization (KHO) algorithm is proposed. Due to the incompleteness of science and technology, there are still some differences in the results [9].

1.4. Innovation. As the current training and monitoring of water dragon boats no longer use manual operations in many fields, the methods usually used tend to be realized by network automatic communication technology, which will make the operating cost relatively high, which is completely unsuitable for the entire water dragon boat training. For the future development of monitoring, it also severely restricts the development of monitoring and monitoring of water dragon boat training. On this basis, a water environment monitoring based on wireless sensor network technology is proposed, which can efficiently and quickly collect all kinds of athletes for dragon boat training. This article is aimed at establishing a hybrid water dragon boat training and monitoring transmission mode by using terminal nodes, cluster head nodes, convergence nodes, and monitoring centers. The water dragon boat training monitoring system designed in this paper has many characteristics: low cost, good scalability, strong reliability, etc., can be used not only in outdoor environments, but also in small monitoring fields such as small homes, and has a very broad application prospect. The entire sensor equipment can monitor the water environment in real time and transmit the collected data to the remote wireless back-end and perform certain analysis and processing at the back-end, which can predict the changing trend of the water body relatively quickly. It can prevent the pollution of the water environment and reduce certain economic losses. Therefore, the main purpose is to comprehensively monitor various parameters in the water dragon boat training monitoring in real time, such as pressure and temperature, to achieve remote monitoring and management. The composition of the wireless sensor network includes many wireless sensor network nodes, and the nodes choose tiny embedded systems. This article is aimed at establishing a hybrid water dragon boat training and monitoring transmission mode by using terminal nodes, cluster head nodes, convergence nodes, and monitoring centers. The data monitored by this system will be more accurate and fast and greatly reduce labor costs, which has application prospects and practical value. This technology has also improved the level of monitoring while providing strong support and provides dragon boat athletes with more scientific and effective data support, allowing them to use more reasonable training methods to strengthen themselves.

\section{Methods and Related Concepts}

2.1. Wireless Sensor Network. The wireless sensor network generally consists of the following three parts: sensor nodes, sink nodes, and task management nodes, as shown in Figure 1.

Use a sufficient number of sensor nodes to be deployed in the area to be monitored (sensor field), to use self-organization to promote it into a complete network system. In this process, the collected monitoring data can be transmitted along other nodes. The whole process is processed by many nodes and then reaches the sink node after multiple hops and then uses the network to transmit to the management node. Users can use the installed host computer software to manage the entire sensor network in a unified manner. It has weak capabilities in data processing, storage, and wireless communication. Generally speaking, some batteries with limited energy are used to power the system. In addition, any node has a certain routing function in addition to the original node terminal function. Nowadays, the software and hardware design of sensor nodes is a very critical link in sensor network technology. The only difference is that the sink node has relatively high requirements for data processing, storage, and wireless communication. Due to the need to connect to an external network, it can realize the communication conversion between two different protocols, and according to the actual instructions given by the user, each node is assigned a different monitoring task, and the information collected by the node is sent to the designated location. Therefore, the convergent sensor node is not only an enhanced function relative to the sensor node but also a management node with computing capabilities; it is also a network device that can communicate, as shown in Figure 2.

The main task is to gather the data and send it to the corresponding PC. Because the existing research does certain data preprocessing at the sink node, such as data deduplication and compression, it cannot be counted as a simple relay. The main function of the sensor module is to collect data and information in the coverage area of the node and to digitize the collected analog data; the function of the processor module is to control the normal operation of the entire wireless node and to perform the data collected by the sensor module. Processing: the main function of the wireless communication module is to realize wireless communication with other nodes, exchange existing control information, and send and receive data; the function of the energy supply module is to provide power.

With the continuous development of sensor networks, the protocol stack of the sensor node is also in the process of continuous improvement. A typical protocol stack model is shown in Figure 3. The model mainly covers five parts: the physical layer, the data link layer, the routing layer, the transmission control layer, and the upper application, which correspond to the five layers in the OSI seven-layer model [10].

The steps of this experiment and the previous experiment are similar, such as the training of the classifier and the calculation of the decision-making reliability; so, the detailed description will not be given. However, it is important to note that because the training sample set is already given, and the classification accuracy of the same type of classifier is fixed. In this experiment, the number of hidden neurons in the $k$ NN classification network is set to 50 . The water training monitoring system is divided into two parts: hardware and software. The hardware is an embedded data acquisition module, which includes acceleration sensor module, heart rate belt, GPRS module, Bluetooth module, and microprocessor [11]. In the software, SOCKET is programmed with TCP/IP protocol to realize data communication, the acceleration sensor and GPS module on the experiment board are programmed to realize data collection, and multiple heart rate measurement nodes are networked and programmed to realize heart rate collection. Data analysis system: the schematic diagram is shown in Figure 4. 


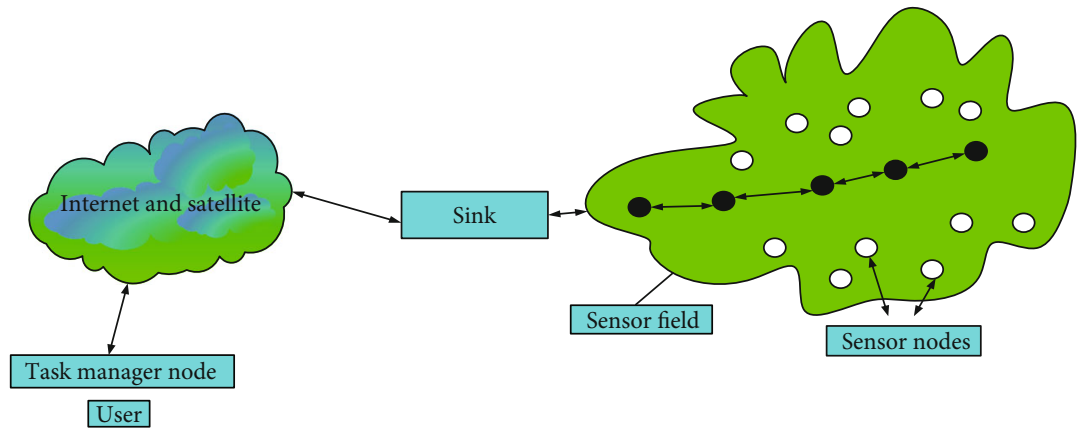

FiguRE 1: Wireless network structure.

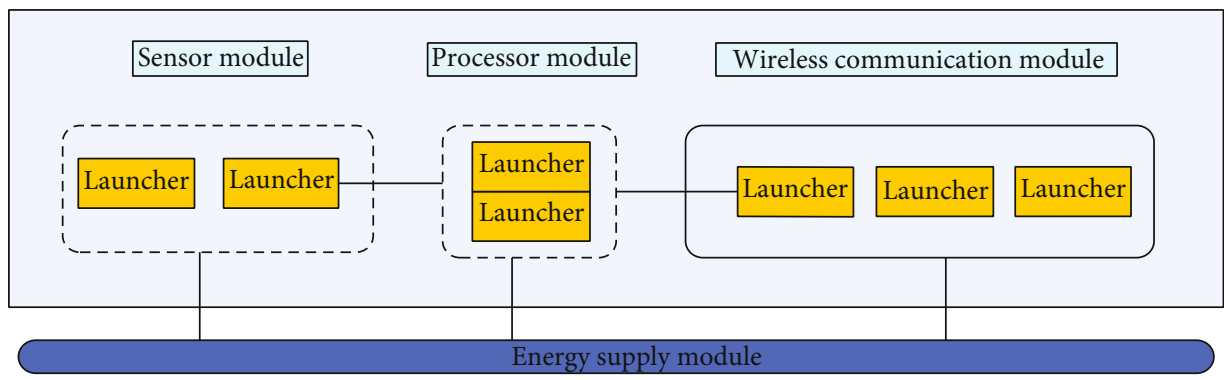

FIGURE 2: Block diagram of wireless sensor nodes.
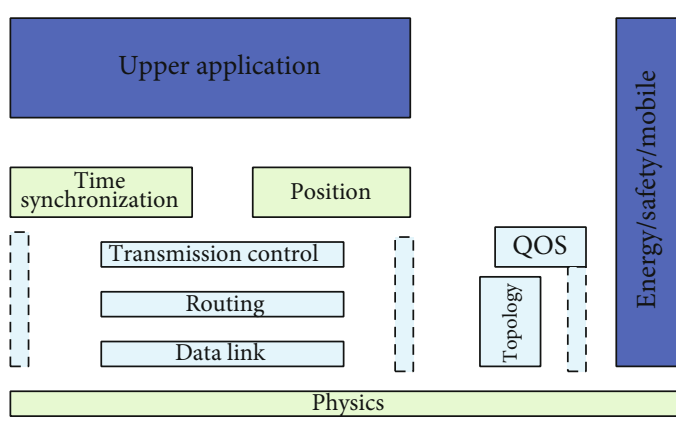

Figure 3: Protocol stack model.

Dragon boat training on the water is a symbol that reflects our national culture and represents the strength and stability of our people, and multiple heart rate belts are, respectively, connected to the Bluetooth host module of the SCM minimal system via Bluetooth. The SCM minimal system controls the Bluetooth host module, acceleration sensor, and GPS module and transmits the processed data to the GPRS module through the serial port, and the GPRS module then transmits the data to the remote computer. Because the data is transmitted through the GPRS network, there is no distance limit when the system is working [12]. The hardware system framework is shown as in Figure 5.

Make full use of the multisensor data resources of different time and spaces, use computer technology to analyze, synthesize, control, and use the multisensor observation data obtained in time series under certain criteria to obtain a consistent interpretation and description of the measured object, then realize the corresponding decision-making and estimation, and make the system obtain more sufficient information than its various components. The system mainly consists of two parts: a data collection subsystem placed in the water and a remote receipt collection subsystem. After that, the data parameters are monitored through the processing, analysis, and storage of the background software. The remote can use the WEB page to query the water dragon boat training parameter index measurement data [13]. Collect the data in the target water area in real time through the data platform, perform real-time statistical analysis, and use big data to push the real-time analysis result data to the analysis department. After the analysis department receives the data, it uses different methods to analyze the indicators, to develop training programs for athletes to help athletes get better results in the competition, as shown in Figure 6.

2.2. Kalman Filter Method. Kalman filter is an algorithm that uses linear system state equations to optimally estimate the system state through system input and output observation data. Since the observation data includes the influence of noise and interference in the system, the optimal estimation can also be regarded as a filtering process. Kalman method includes the state space method and projection method. Kalman added a concept to the method the state of the system and introduced two mathematical models state and observation, that is, the classical state equation and observation equation. The true value is inaccessible, and it can only be based on the minimum mean square error to make the 


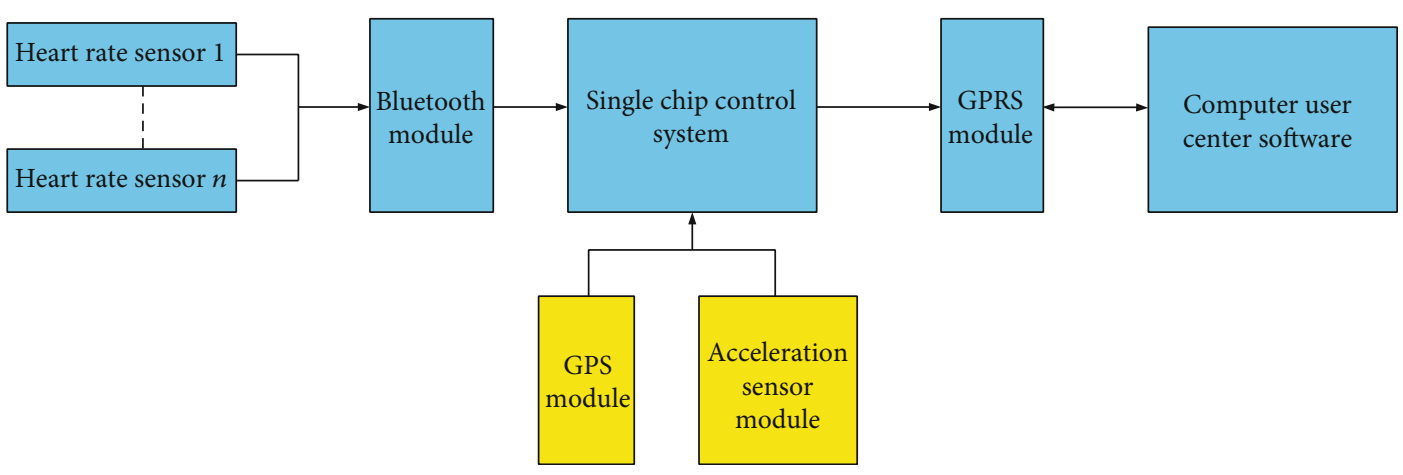

FIgURE 4: Schematic diagram of water training monitoring.

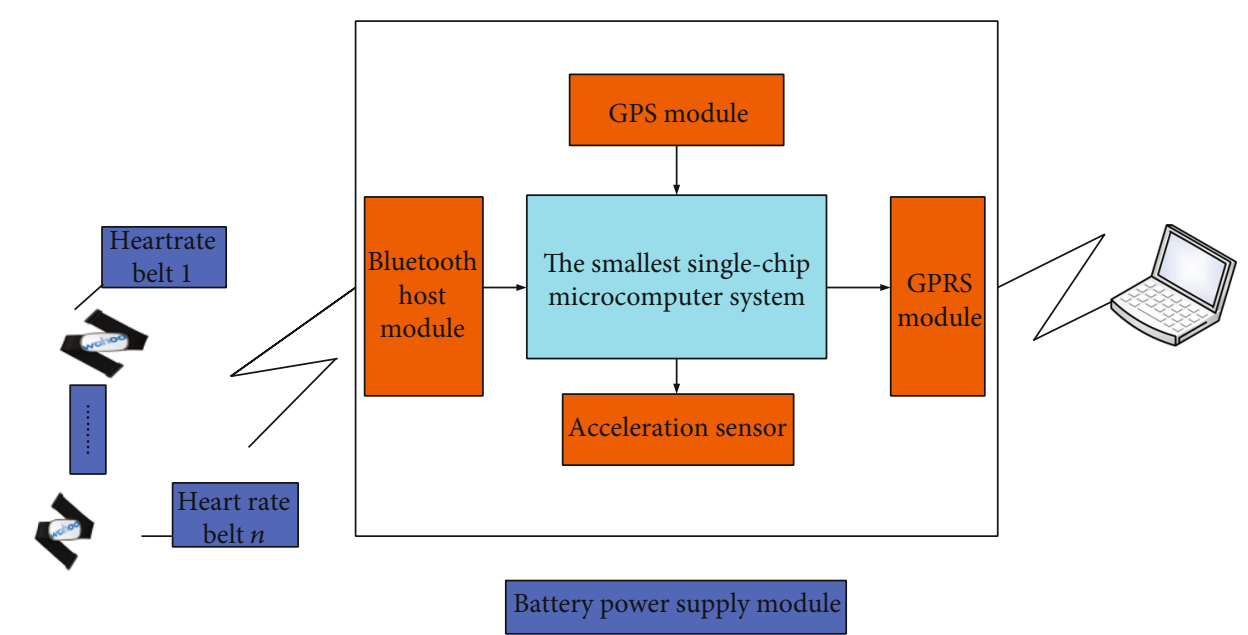

FIGURE 5: Hardware system framework diagram.

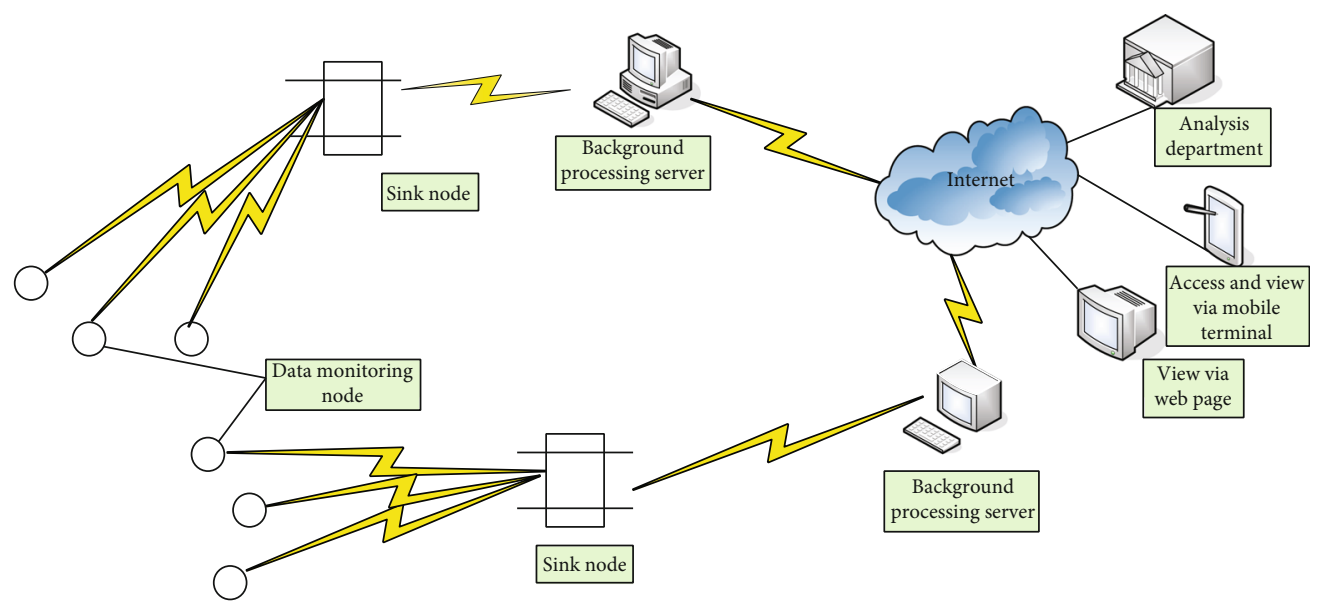

FiguRe 6: Data analysis system.

estimated value as close to the true value as possible, through the input and output observation data of the system, the algorithm for optimal estimation of the system state. Since the observation data includes the influence of noise and interference in the system, the optimal estimation can also be regarded as a filtering process. On this basis, he used the projection method to propose the optimal and Kalman filter equations [14]. The biggest advantage of Kalman filtering is that filtering is a state-space method in the time domain, which has obvious intuitive meaning. At the same 
time, the Kalman filter method is a recursive method, so that the fusion system does not need to store large data and perform complex calculations and can be conveniently used in real time on a computer [15]. Kalman filtering is suitable for filtering problems in stationary or nonstationary random processes, as well as filtering problems in time-varying systems and multivariable systems.

The specific equation of Kalman filter is as follows:

$$
x(t+1)=b(t) x(t)+g(t) u(t)+w(t)
$$

In the above formula, we can see that $X(k)$ represents an $n$-dimensional vector, which represents the target state at time $t$, which is called the state equation; $\beta(t)$ is the $n \times n$ order state transition matrix at time $t, U(t)$ is the $m$-dimensional input vector, $G(t)$ is the $n \times m$-order input matrix, and $W(t)$ is the $n$-dimensional process noise, which conforms to the Gaussian white noise distribution, and $E[W(\mathrm{t})]=0, E\left[W(\mathrm{t}) W^{T}(J)\right]=Q(K) \eta_{T J}$.

Among them, they satisfy

$$
h_{t j}=\left\{\begin{array}{l}
0, t^{\dot{i} j} \\
1, t=j
\end{array} .\right.
$$

The sensor observation equation used for target tracking is

$$
z(t)=h(t) x(t)+v(t)
$$

$Z(t)$ is a $p$-dimensional vector, which represents the measurement vector at time $t, H(t)$ is a $p \times n$-order measurement matrix, $V(t)$ represents $p$-dimensional measurement noise, which conforms to the Gauss white noise distribution, and have

$$
\left\{\begin{array}{l}
E[V(\mathrm{t})]=0, \\
E[V(\mathrm{t})] V^{T}(J)=R(\mathrm{t}) \eta_{\mathrm{tj}} .
\end{array}\right.
$$

If the filtered value $x^{U}(t / t)$ and the covariance $P(t / t)$ of the target observation state $X(t)$ at time $t$ are known, then the Kalman filter method can be used to obtain the state prediction value of the moving target at time $t+1$ :

$$
\stackrel{U}{x}(t+1 / t)=j(t) \stackrel{U}{x}(t / t)+g(t) u(t) .
$$

Forecast covariance matrix is as follows:

$$
p(t+1 / t)=j(t) p(t / t) j^{t}+q(t)
$$

Innovation covariance matrix is as follows:

$$
s(t+1)=h(t+1) p(t+1 / t) h^{t}(t+1)+r(t+1) .
$$

Gain matrix is as follows:

$$
k(t+1)=P(t+1 / t) h^{t}(t+1) s^{-1}(t+1) .
$$

State filter value is as follows:

$$
\stackrel{U}{x}(t+1 / t+1)=\stackrel{U}{x}(t+1 / t)+k(t+1)[z(t+1) \stackrel{U}{z}(t+1 / t)] .
$$

The filter covariance matrix is

$$
p(t+1 / t+1)=p(t+1 / t)-k(t+1) s(t+1) k^{t}(k+1) .
$$

Two of the more significant problems are as follows: first, when the combined data of multiple sensors is redundant, the amount of calculation will increase drastically by the geometric exponent of the filter dimension, which will make the real-time performance difficult to meet; the second is the number of sensors. Increasing generally increases the possibility of failure. When a sensor fails and the system is not found in time, the failure will affect the entire system and reduce the reliability of the system.

2.3. Bayesian Estimation Method. The installation position of the multisensor is also one of the important indicators that affect the data. How to combine the multisensor to play the best value of each part has great guiding significance for the results of the experiment. Bayesian estimation is a main method to fuse low-level multisensor data in static environments. This method is to recombine multisensor data according to probability and use conditional probability to express the measurement uncertainty. If the observation coordinates of each sensor are the same, and then the measurement data obtained from the sensors can be fused by the direct method [16]. However, most of the time, the measurement data of different sensors come from different coordinate systems. At this time, we should use indirect methods to perform Bayesian estimation and fusion of the measurement data of different sensors. Assuming that $p\left(x_{i} \mid x\right)$ is the conditional probability that the measurement is $x_{i}$ under the condition that the true value is $X$, it is a known quantity. When the received measurement data is $x_{i}$, the posterior probability of the true value, $X$ is calculated by the Bayes formula as

$$
p\left(x \mid x_{i}\right)=\sum_{y}^{p\left(x_{i} \mid x\right) p(x)} p\left(x_{i} \mid y\right) p(y)
$$

Assuming that the estimated value of the unknown $\theta$ is $\theta^{\wedge}$, a commonly used estimation method is to take the mean of the posterior distribution, that is to say

$$
\stackrel{U}{q}=\stackrel{U}{q}\left(x_{1}, x_{2}, \cdots, x_{n}\right)=E\left(q \mid x_{1}, x_{2}, \cdots, x_{n}\right)
$$

It can be seen from the above definition that the posterior distribution depends on the sample; so, the estimation of the unknown parameter must be related to the sample selection. In this estimation method, the estimated value of $\theta^{\wedge}$ is generally the average of the parameters of the posterior distribution. Another method that can be used to calculate the estimated value is the method with the smallest deviation, when he meets 


$$
E\left((\theta-\alpha)^{2} \mid X_{1}, X_{2}, \cdots, X_{N}\right) .
$$

If the minimum value of the above formula is, that is, the deviation of each parameter is the smallest. To minimize the deviation, the following formula can be derived, namely,

$$
\operatorname{se}\left((\theta-\alpha)^{2} \mid x_{1}, x_{2}, \cdots, x_{n}\right)=2 e\left(\theta \| x_{1}, x_{2}, \cdots, x_{n}\right)-2 \alpha=0 .
$$

Assuming that B1, B2, ... are several possible premises of a certain process, then $P(\mathrm{Bi})$ is the people's estimate of the probability of each premise condition in advance, which is called the prior probability. If this process yields a result $\mathrm{A}$, then the Bayesian formula provides a way for us to make a new evaluation of the preconditions based on the appearance of A. The above transformation can be obtained:

$$
\alpha=\theta=e\left(\theta \mid x_{1}, x_{2}, \cdots, x_{n}\right) .
$$

Bayesian decision-making is to estimate part of the unknown state with subjective probability under incomplete intelligence, then use Bayesian formula to correct the probability of occurrence, and finally use the expected value and revised probability to make the optimal decision.

2.4. D-S Evidence Reasoning Method. The Dempster-Shafer (D-S) method of evidence reasoning was first proposed by Dempster and developed by Shafer.

The method belonging to the theory of imprecise reasoning can be regarded as an extension of the Bayesian method. Compared with Bayesian estimation, which requires a priori probability, D-S evidence reasoning can solve the uncertainty caused by ignorance. In a data fusion system composed of multiple sensors, each sensor can give a set of data (or a set of evidence or propositions), so that the corresponding mass distribution function can be established [17]. Here, each data source can be regarded as a recommendation degree. Under the same conditions, after combining different evidence bodies by the Dempster combination rule, a brand-new recommendation degree is calculated, and then the credibility of this recommendation degree is calculated, and then through a certain screening rule, the final result is shown in Figure 7.

The D-S evidence reasoning method uses a recognition framework to represent the collection that needs to be fused, and it gives such a function:

$$
m(\Theta)=0, \sum_{\mathrm{a} \subset \Theta} m(a)=1 .
$$

For any set, D-S, evidence reasoning gives a concept of credibility function:

$$
\operatorname{bel}(a)=\sum_{\mathrm{b} c \mathrm{a}} m(b) .
$$

However, it is still not enough to express the credibility of each set A with only one credibility function, because Bel (A) does not reflect the degree of suspicion for this set
A [18]. Based on this, if we want to describe the credibility of a set A more comprehensively, we need to add a quantity that can reflect the degree of suspicion A. Then, this definition can be given:

$$
\begin{aligned}
& \operatorname{pl}(a)=1-\operatorname{bel}(a), \\
& \operatorname{dou}(a)=\operatorname{bel}(a) .
\end{aligned}
$$

We can clearly derive the fusion formula of multiple credibility; first, the following hypothesis needs to be given: $m_{1}, m_{2}, \cdots, m_{n}$ is used to represent the credibility distribution function of $n$ data, and these $n$ data are independent of each other; then, the credibility function after fusion can be written in the following form:

$$
m(a)=m_{1} \AA m_{2} \AA \cdots \AA m_{n}=1-\sum_{a_{i} C b_{i}=Q}^{\sum_{a_{i} C b_{i}=a} m_{1}\left(a_{i}\right) m_{2}\left(b_{i}\right)} m_{1}\left(a_{i}\right) m_{2}\left(b_{i}\right) .
$$

\section{Comparative Analysis Experiment}

3.1. Experimental Design. The experiment was carried out in the lake where water dragon boat competitions have been held over the years. Related experimental equipment was arranged on the shore. Video monitors and sensors were installed on the dragon boat page and some parts of the athletes. Multisensor data fusion is an emerging research field, which is a research on data processing aimed at the specific problem of using multiple sensors in a system. Multisensor data fusion technology is a practical application technology developed in recent years. It is a new technology that intersects multiple disciplines. It involves signal processing, probability and statistics, information theory, pattern recognition, artificial intelligence, fuzzy mathematics, and other theories. In this experiment, the main purpose is to verify the performance of the three fusion methods proposed in this paper, RP fusion and NB and WMV fusion rules, under the conditions of different numbers of classifiers and different classification accuracy. Because in the actual classification application, the performance of the local classifier is fixed; so, the use of variable data sets can better study the impact of the accuracy of different classifiers on the performance of the fusion algorithm. In this experiment, we use the Gaussian function to randomly generate a data set. The number of target category labels is set to 5. Each sample data consists of two randomly generated attributes that conform to different Gaussian distributions, and the two attributes are independent of each other. As shown in Table 1, the standard deviation of the two attributes of the sensor can be changed by the variable $\alpha$. The larger the parameter $\alpha$, the larger the standard deviation of the two attributes. For example, the following two probability density functions $x_{1}\left|\omega_{3} \sim n(30,4 \alpha), x_{2}\right| \omega_{3} \sim n(10,4 \alpha)$, respectively, represent the distribution of the two attributes of the category label $\omega_{3}$. Obviously, the classification performance of the classifier is determined by $\alpha$. The larger the $\alpha$, the less concentrated the data distribution of each category, and the overlapping range will increase accordingly; so, the classification accuracy of the sensor will be smaller. Therefore, the 


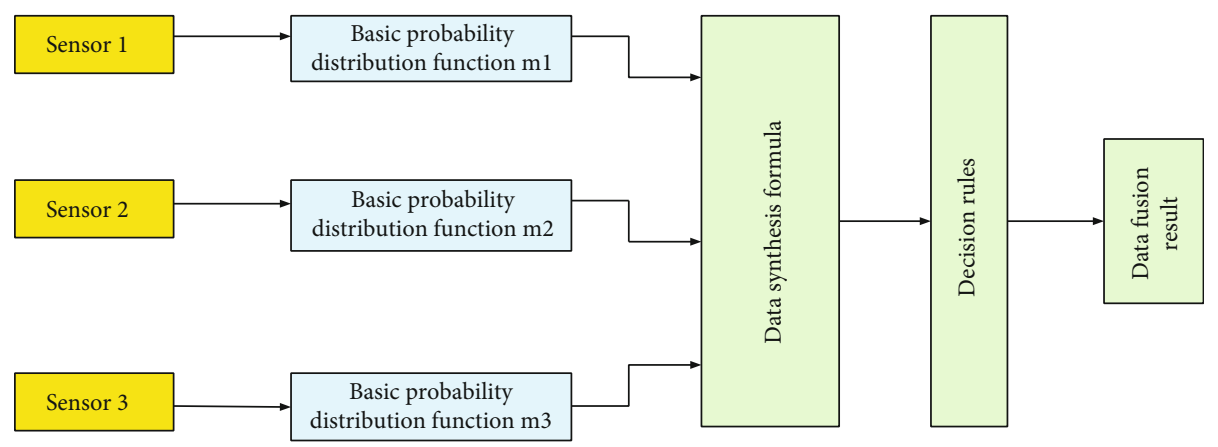

Figure 7: Flow chart of multisensor data fusion.

TABLE 1: Randomly generated data.

\begin{tabular}{lllc}
\hline Category & $\mu 1$ & $\mu 2$ & $\beta$ \\
\hline w1 & 20 & 10 & $3 \alpha$ \\
w2 & 10 & 20 & $5 \alpha$ \\
w3 & 15 & 30 & $10 \alpha$ \\
w4 & 15 & 30 & $9 \alpha$ \\
w5 & 20 & 20 & $7 \alpha$ \\
\hline
\end{tabular}

classification performance of the sensor can be adjusted by the parameter $\alpha$ as shown in Table 1 .

From the data in the above table, it can be found that the data set is mainly concentrated in the interval of 10-20, and the overlapping range can be established by using the Gaussian function. Gaussian function is widely used in statistics to describe the normal distribution.

As shown in Figure 8, it represents a data set randomly generated by the Gaussian function when $\alpha$ is equal to 1 , and the sample data is equal to 100 . In the actual experiment, the number of samples used to train the sensor is set to 300. At the same time, to prevent the one-sidedness of the simulation results, in each round of experiments, a new data set will be randomly generated. Considering the reliability of the experimental results, the experiment was repeated 20 times, and the classification decision result was obtained each time. The average classification decision result of 20 times was calculated to obtain the average classification accuracy. To obtain the classification performance of each sensor, 1500 training sample sets and 500 test sample sets were randomly generated in each experiment. And the number of training samples and test samples of each category in each experiment is the same, that is, 300 and 100, respectively. After the classifier is trained, another 1000 observations are generated, and the corresponding number of each category is about 200. Then, using the trained classifier to classify and make decisions on these data to obtain local decisions, and at the same time obtain the reliability of the classifier's decision, the fusion center then conducts decision fusion based on the local decision and decision reliability [19].

3.2. Experimental Method. Aiming at the problem of slow paddle frequency in the start and sprint phases that cannot keep up with the rhythm, the training method is mainly short-stroke training, which is high-frequency rapid strokes of 10 paddles, 20 paddles, and 30 paddles; supplemented by

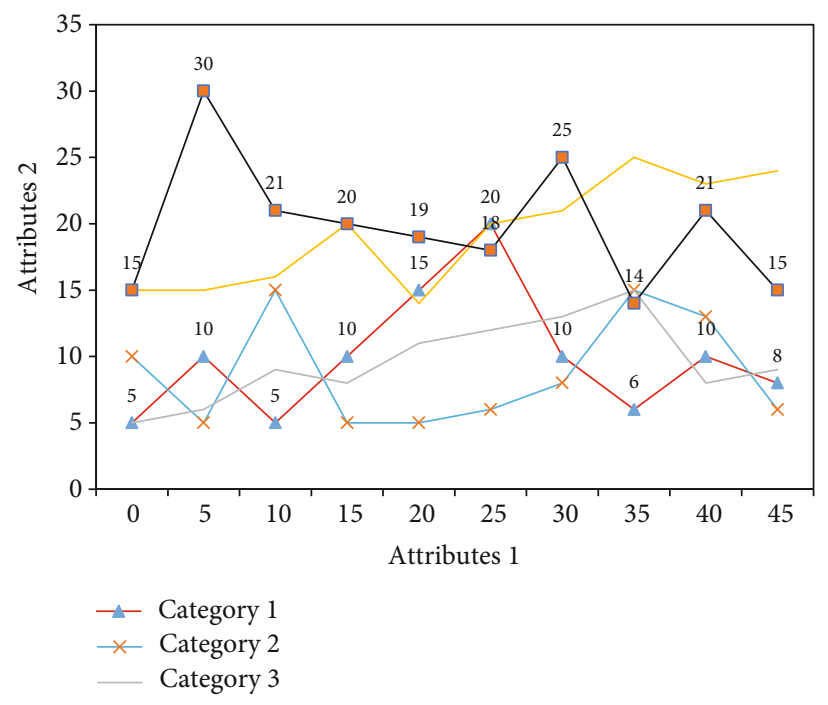

Figure 8: Example of generating sample data.

functional training, vibration rods, and power ropes, barbells and other equipment can be used for upper limb speed endurance training. This experiment will use the actual dragon boat recognition data set collected during the monitoring of the water dragon boat training project for simulation. In the monitored project, a total of 23 sensor nodes are arranged on the roadside to collect data on dragon boats and athletes [20]. When a dragon boat passes by, the sensor will collect three types of data: infrared, earthquake, and sound of the dragon boat. Perform segmentation and feature extraction on the collected data. In the actual experiment, 11 sensor-node pairs will be selected for classification. The experiment used sound and seismic signals. After fast Fourier transform, the characteristics of each signal are proposed. Each has 297 characteristics, and the length of each characteristic is 50 values between 0 and 1 . The data obtained were measured using a logistic regression model, considering the different data of each individual of each athlete, and combining these variables for data screening.

\section{Analysis of Experimental Results}

4.1. Comparative Analysis. The experimental results obtained are shown in the following figure. The classification accuracy of the fusion result is defined as the proportion of correctly identified targets in each classification. The RP fusion rule 


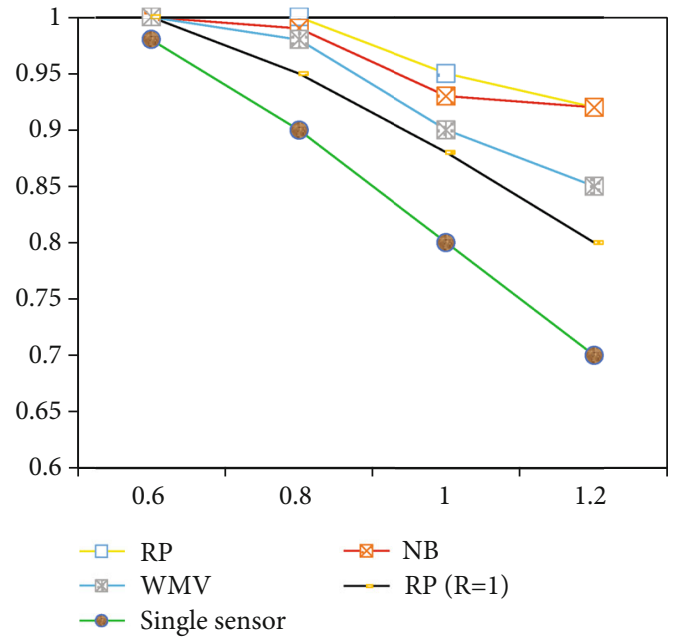

(a)

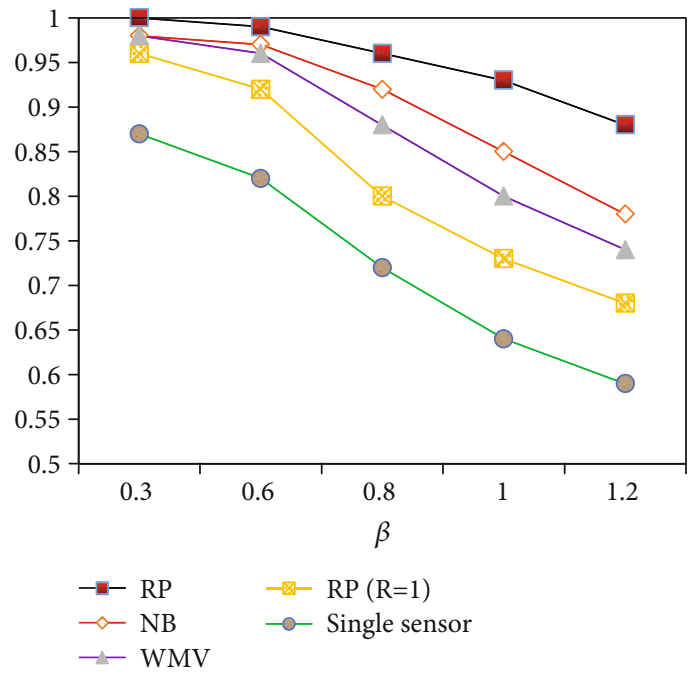

(b)

FIgURE 9: (a) When the local classifier is $k$-NN, the accuracy of the fusion results of each fusion rule varies $\beta$. (b) When the local classifier is ELM, the accuracy of the fusion results of each fusion rule varies $\beta$.

proposed in this paper is compared with the naive Bayes fusion rule and the fusion performance of WMV Considering the local classification decision in $k$-NN and ELM neural network, and the average classification accuracy of the fusion results of these three fusion methods (plus and minus one standard deviation) varies with the parameter $\beta$ and the classifier number of changes, as shown in Figure 9 below.

The changes are shown in Figure 9. Figures 9(a) and 9 (b), respectively, show that when the number of sensors is fixed at 5 , the fusion accuracy of each fusion rule changes with $\beta$ when the classifier is $k$-NN and ELM neural network case. It can be seen from Figure 9(a) that when the value of the parameter increases from 0.97 to 2.5 , the average classification accuracy of the $k$-NN classifier decreases from 0.97 to 0.4 , and the accuracy of the fusion results of the three fusion rules is also correspondingly, but the RP fusion rule proposed in this paper is still better than the other two fusion rules. In the simulation diagram (b), as the parameter $\beta$ increases, the classification performance of the local classifier decreases accordingly, and the accuracy of the fusion results of each fusion rule changes with $\beta$ to be roughly the same as the diagram (a). However, it can be seen from Figures 9(a) and 9(b) that the performance of the $k$-NN classifier is better than the ELM classifier, especially when the value of $\beta$ is in the interval [0-1.4]. Therefore, the accuracy of the final fusion result obtained by using the $k$-NN classifier for local classification decision is higher than the case of using the ELM classifier [21]. Clearly, from the simulation results, it can be verified that the reliability-probability fusion rule proposed in this paper can more effectively improve the classification accuracy, and the performance is always better than the NB fusion rule and WMV fusion rule, especially when the local classifier classification performance when it is poor, the RP fusion rule proposed in this paper to fuse the classification decision results of multiple classifiers with poor classification performance, and the accuracy of the final fusion result can be greatly improved.
4.2. The Accuracy of the Fusion Result Varies with the Number of Sensors. The changes are shown in Figure 10. Figures 10(a) and 10(b) show how the accuracy of the fusion result varies with the number of sensors when the local classifiers arek -NN and ELM, respectively. At this time, $\beta=1.5$ is fixed during the simulation process. Like the conclusions drawn in Figures 9(a) and 9(b), the experimental results still prove that the final fusion result obtained by the RP fusion rule proposed in this paper is more accurate than the NB fusion rule and WMV fusion rule. At the same time, the analysis shows that when the number of sensors is increased from 1 to 7 , and the accuracy of the fusion result increases quickly compared with a single sensor. However, when the number of sensors is greater than 7, the accuracy of the final fusion result is the effect of increasing degree gradually decreases. In actual target classification applications, when a certain fusion accuracy is achieved, the number of sensors involved in monitoring should be minimized to reduce costs. Finally, it can be seen that the fusion rule proposed in this paper is very similar to the NB fusion rule, but the decision-making performance of the two is different. From the above simulation results, it can be concluded that when the decision reliability $R=1$ is set, the performance of the RP fusion rule is much lower than the other two types of fusion algorithms [22]. This shows that the calculation method of decision-making reliability is a key factor that affects the accuracy of RP-based fusion. Therefore, in practical applications, it is necessary to design a reasonable reliability calculation method for different application scenarios, and it will be possible to obtain better fusion performance [23].

4.3. When the Classifier Is ELM, the Fusion Accuracy Varies with the Number of Sensors. The change is shown in Figure 11. From Figure 11(a), it can also be concluded that when the classifier is $k$-NN, the three fusion rules increase with the number of sensors, and the accuracy of the fusion result is also improved accordingly. Before the improvement, the highest value of a 


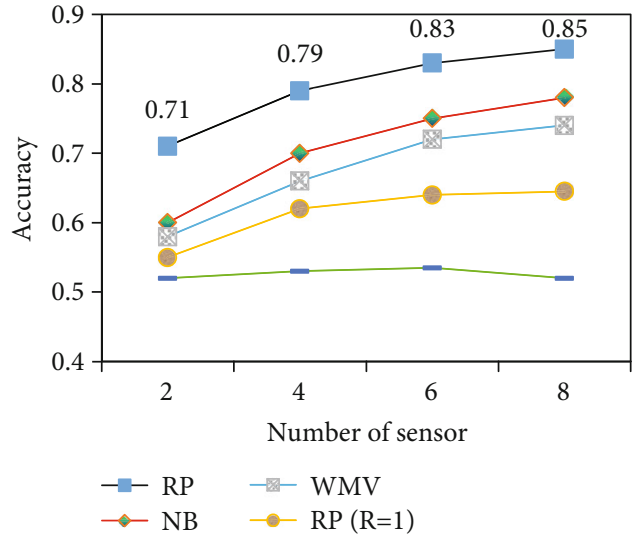

(a)

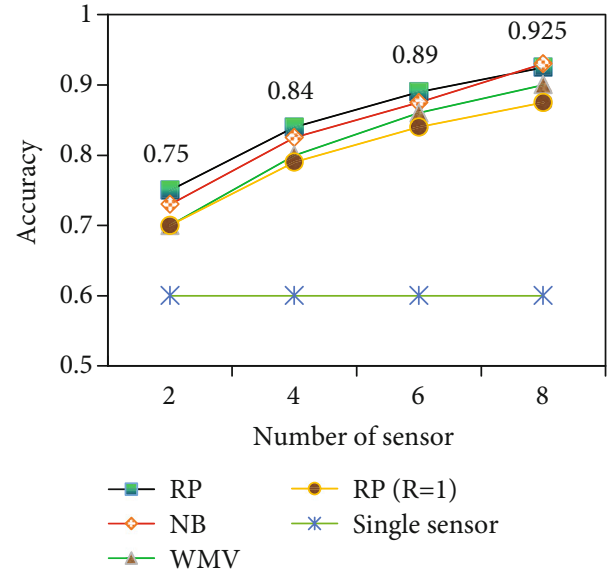

(b)

FIGURE 10: (a) When the local classifier is $k$-NN, the accuracy of the fusion results of each fusion rule varies with the number of sensors. (b) When the local classifier is ELM, the accuracy of the fusion results of each fusion rule varies with the number of sensors.

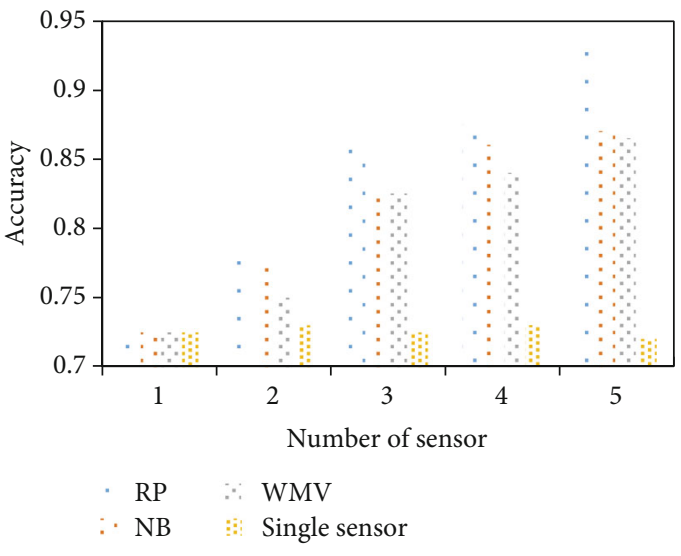

(a)

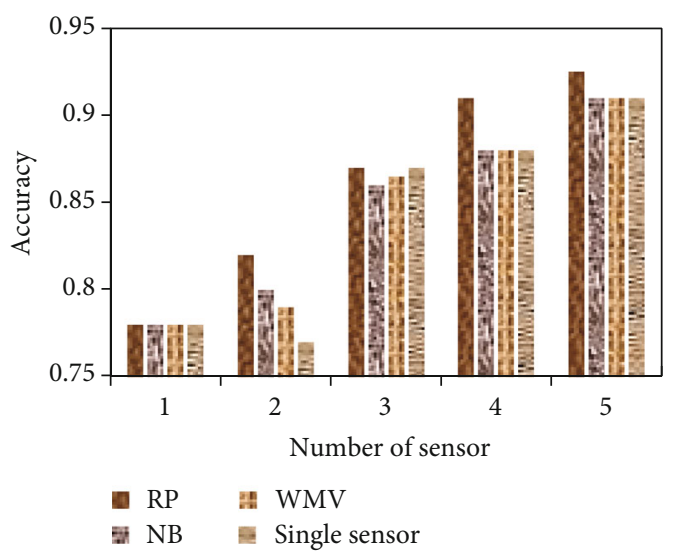

(b)

FIgURE 11: (a) When the classifier is $k$-NN, the accuracy of the fusion results of each fusion rule varies with the number of sensors. (b) When the classifier is ELM, the accuracy of the fusion results of each fusion rule varies with the number of sensors.

single sensor is 0.73 ; after the improvement, the highest value of a single sensor can reach 0.91 ; and the accuracy rates of $R P, N B$, and WMV have been greatly improved. However, the final fusion accuracy obtained by the RP fusion rules proposed in this paper is always higher than that of the NB fusion rule and WMV fusion rule [24]. The simulation result of analysis diagram (b) can still be obtained. When the classifier is ELM, the classification accuracy of the decision results of multiple classifiers through the fusion processing of the three fusion rules is clearly more accurate than that of a single sensor. However, the RP fusion rule performance is still better than the other two commonly used fusion rules; so, the simulation results prove that the RP fusion rule proposed in this paper has superior performance, and the decision fusion accuracy is higher than that of the NB and WMV fusion rules. From the simulation results, it can also be found that, different from the previous experimental results, the final decision accuracy of the NB fusion algorithm and WMV fusion algorithm at this time is not much different, indicating that the stability is poor due to the limitation of the application scenario, and in this article, the proposed decision fusion algorithm is more stable in improving the classification accuracy.

\section{Conclusions}

Bayesian estimation provides a means for data fusion and is a common method for fusing high-level information from multiple sensors in a static environment. It enables sensor information to be combined according to the principle of probability, and the measurement uncertainty is expressed in conditional probability. Based on the sports training theory and combined with the special characteristics of the competitive dragon boat event, a series of comparative analysis of different training methods and methods aimed at improving the special endurance training of the upper limbs of competitive dragon boat athletes. Indepth study of the current status of the special endurance training of the upper limbs of Chinese competitive dragon boat athletes determines the existing 
problems and analyzes them. The water training monitoring system based on multisensor data fusion technology has the characteristics of high sampling frequency and strong processing ability. It can convert the collected heart rate, speed, acceleration, and other data into images for real-time display and monitor and analyze training effects. The system uses modern science and technology such as GPRS network transmission, paddle frequency calculation, embedded system, and GPS, without affecting the training of athletes, collects sports information and instantly generates monitoring process diagrams of sports training parameters, enabling coaches and athletes clearly understand the real-time kinematic parameter information in the training process, provides a scientific basis for coaches to diagnose training effects in real time, improves the efficiency of athletes' special training, and conducts targeted training. Under various methods of fusing data, we have performed a certain analysis on the data transmitted by this sensor, including the heart rate of dragon boat athletes, and what the speed of the dragon boat is at what heart rate. Understand that with our help, athletes can make their training more effective. Since the athlete's physical fitness and other factors have not been fully investigated when selecting the samples, the experimental data will still be a little biased. Weather factors and other factors will affect the parameters of the game, so the content involved in this research is too much. Monotonous, more detailed research will be carried out next.

\section{Data Availability}

Data sharing not applicable to this article as no datasets were generated or analyzed during the current study.

\section{Conflicts of Interest}

There is no potential conflict of interest in our paper.

\section{Authors' Contributions}

The author has seen the manuscript and approved to submit to your journal.

\section{Acknowledgments}

This work was supported by Huazhong University of Science and Technology Independent Innovation Research Fund (Humanities Social Sciences) (2019WKYXQN003).

\section{References}

[1] F. Xiao, "Multi-sensor data fusion based on the belief divergence measure of evidences and the belief entropy," Information Fusion, vol. 46, pp. 23-32, 2018.

[2] M. Elhoseny, "Multi-object detection and tracking (MODT) machine learning model for real-time video surveillance systems," Circuits, Systems, and Signal Processing, vol. 39, no. 2, pp. 611-630, 2020.

[3] J. Zhang and L. Du, "Research on industrial internet of things system based on multisensor data fusion," Boletin Tecnico/ Technical Bulletin, vol. 55, no. 16, pp. 457-462, 2017.
[4] F. Wu, X. Liu, and Y. Wang, "Research on software design of intelligent sensor robot system based on multidata fusion," Journal of Sensors, vol. 2021, no. 20, p. 10, 2021.

[5] Z. Zhang and L. I. Fang, "Research on the water pollution monitoring and rapid decision-making system based on artificial intelligence agent.," Journal of Environmental Protection and Ecology, vol. 20, no. 3, pp. 1565-1573, 2019.

[6] R. G. Ma, Z. H. Ma, and Y. X. Wang, "Study on positioning technology of ,mileage piles based on multi-sensor information fusion," Journal of Highway \& Transportation Research \& Development, vol. 10, no. 4, pp. 7-12, 2016.

[7] X. Xu, D. Cui, Y. Li, and Y. Xiao, "Research on ship trajectory extraction based on multi-attribute DBSCAN optimisation algorithm," Polish Maritime Research, vol. 28, no. 1, pp. 136148, 2021.

[8] N. Zhang and F. Li, "Research on safety monitoring and warning system based on information fusion for coking production," International Journal of Hybrid Information Technology, vol. 9, no. 8, pp. 303-314, 2016.

[9] H. Cui, X. Dong, H. Deng, M. Dehghani, K. Alsubhi, and H. M. Aljahdali, "Cyber attack detection process in sensor of DC micro-grids under electric vehicle based on Hilbert-Huang transform and deep learning," IEEE Sensors Journal, vol. 21, no. 14, pp. 1-1, 2021.

[10] H. S. Zhai, Z. Y. Wen, and H. P. Yu, "New method of multisensor data fusion based on multiscale analysis and UKF," International Journal of Control \& Automation, vol. 9, no. 2, pp. 329-342, 2016.

[11] S. Ji, Z. Y. Chen, P. Guo et al., "Bayesian approach for multisensor data fusion based on compressed sensing for wireless structural damage signal," Journal of Internet Technology, vol. 17, no. 7, pp. 1363-1371, 2016.

[12] X. Ouyang, W. He, W. Lu, and Z. Bian, "Study on 3D border surveillance system and multi-sensor information fusion technology," Bandaoti Guangdian/Semiconductor Optoelectronics, vol. 39, no. 2, pp. 298-304, 2018.

[13] X. H. Wu and S. M. Song, "Covariance intersection-based fusion algorithm for asynchronous multirate multisensor system with cross-correlation," Iet Science Measurement \& Technology, vol. 11, no. 7, pp. 878-885, 2017.

[14] S. Kumar and V. K. Chaurasiya, "A multisensor data fusion strategy for path selection in internet-of-things oriented wireless sensor network (WSN)," Concurrency and Computation: Practice and Experience, vol. 30, no. 18, pp. e4477.1e4477.14, 2018.

[15] C. Yang, J. Guo, L. Zhang, and Q. Chen, "Multi-sensor faulttolerant fusion algorithm using unscented information filter," Journal of Nanjing University of ence and Technology, vol. 41, no. 3, pp. 269-277, 2017.

[16] C. Bachmann, B. Abdulhai, M. J. Roorda, and B. Moshiri, "Multisensor data integration and fusion in traffic operations and management," Transportation Research Record, vol. 2308, no. 1, pp. 27-36, 2018.

[17] P. Braca, R. Goldhahn, G. Ferri, and K. D. LePage, "Distributed information fusion in multistatic sensor networks for underwater surveillance," IEEE Sensors Journal, vol. 16, no. 11, pp. 4003-4014, 2016.

[18] H. K. Lee, S. G. Shin, and D. S. Kwon, "Design of emergency braking algorithm for pedestrian protection based on multisensor fusion," International Journal of Automotive Technology, vol. 18, no. 6, pp. 1067-1076, 2017. 
[19] Z. Yu, L. Chang, and B. Qian, “A belief-rule-based model for information fusion with insufficient multi-sensor data and domain knowledge using evolutionary algorithms with operator recommendations," Soft Computing, vol. 23, no. 13, pp. 5129-5142, 2019.

[20] J. W. Hu, B. Y. Zheng, C. Wang et al., "A survey on multisensor fusion based obstacle detection for intelligent ground vehicles in off-road environments," Frontiers of Information Technology \& Electronic Engineering, vol. 21, no. 5, pp. 675692, 2020.

[21] K. Da, T. Li, Y. Zhu, H. Fan, and Q. Fu, "Recent advances in multisensor multitarget tracking using random finite set," Frontiers of Information Technology \& Electronic Engineering, vol. 22, no. 1, pp. 5-24, 2021.

[22] M. Mosalanejad and M. M. Arefi, "UKF-based soft sensor design for joint estimation of chemical processes with multisensor information fusion and infrequent measurements," Science, Measurement \& Technology, IET, vol. 12, no. 6, pp. 755763, 2018.

[23] A. Rubaiyat, Y. Fallah, X. Li, G. Bansal, and T. Infotechnology, "Multi-sensor data fusion for vehicle detection in autonomous vehicle applications," Electronic Imaging, vol. 2018, no. 17, pp. 257-1-257-6, 2018.

[24] L. Wang, L. Liang, D. Ma, H. Wang, and X. Liu, "Localization method of biped robot based on multi-sensor information fusion," Zhongguo Guanxing Jishu Xuebao/Journal of Chinese Inertial Technology, vol. 26, no. 5, pp. 629-634, 2018. 